\title{
Psoas abscess as a complication of subclavian venous catheterization
}

\author{
T. Kwok and J. Coles \\ Department of Geriatric Medicine, St George's Hospital, London SW17 ORE, UK.
}

\begin{abstract}
Summary: A 76 year old woman developed a psoas abscess from vascular seeding of an infected subclavian venous catheter. The presentation was insidious and diagnosis delayed. Despite adequate surgical drainage, the case proved to be fatal. Venous catheters should be regarded as a potential source of septicaemia and occult abscess formation.
\end{abstract}

\section{Introduction}

Psoas abscess is uncommon and is easily missed clinically. With decreasing prevalence of tuberculosis, pyogenic bacteria have become the commonest infecting agents. The source of infection is commonly from adjacent structures such as the gastrointestinal tract and the axial skeleton. Primary psoas abscess is rare. Staphylococcus is the commonest aetiological organism and a haematogenous origin is most likely. There has been one reported case of psoas abscess resulting from vascular access (polytetrafluoroethylene fistula) in a patient on chronic haemodialysis. ${ }^{1}$ A case of psoas abscess as a complication of an infected subclavian venous catheter is reported.

\section{Case report}

A 76 year old woman was admitted to an intensive care unit in September 1988 after a major paracetamol overdose. On admission, she was unconscious, in shock and acidotic. She was treated with acetylcysteine, gastric lavage and charcoal, and dopamine infusion via a subclavian venous catheter. She regained consciousness the following day but was noted to be confused and withdrawn. She was then transferred to a general medical ward.

On the third day, a skin abscess developed at the site of the subclavian line. The catheter was promptly removed and a course of oral flucloxacillin was started. The catheter tip grew Staphylococ-

Correspondence: T. Kwok, Department of Medicine for the Elderly, Leicester General Hospital, Gwendolen Road, Leicester LE5 4PW, UK.

Accepted: 2 May 1990 cus aureus which was sensitive to flucloxacillin. Blood culture was not taken. Meanwhile her mental state remained unchanged. The history taken from her sister and her general practitioner was suggestive of depression prior to overdose. She was then suspected to be depressed and was transferred to a psychogeriatric ward. Despite a course of twelve electroconvulsive therapy treatments and antidepressants, she remained withdrawn and uncommunicative. She had a computed tomographic head scan which showed a right parietal cerebral infarct, which was presumed to be secondary to the initial hypotensive episode and contributory to her mental state. Because of poor oral intake, nasogastric feeding was later instituted. Nevertheless her weight continued to fall and she became increasingly weak.

During the first 5 weeks in the psychogeriatric ward, she was noted to have recurrent pyrexia and complained of left loin pain episodically. She was treated for urinary tract and lower respiratory tract infection with three different courses of antibiotics (co-trimoxazole, ampicillin and erythromycin). The diagnoses were supported by either positive urine culture or lung shadowing on chest X-ray. Six blood cultures taken from different venous sites during the three pyrexial episodes were all negative, though the samples were taken no more than 3 days after the preceding antibiotics were stopped. During a later episode of pyrexia in December, blood culture grew $S$. aureus. By then she was cachectic and bedbound. She was transferred to a geriatric ward for further investigation.

Because of abnormal liver function tests, an abdominal ultrasound scan was performed. It showed a left psoas abscess, fatty liver and pus in the bladder. A small amount of thick pus was aspirated from the abscess under ultrasound con- 
trol. An abdominal computed tomographic (CT) scan showed that the abscess extended the whole length of the left psoas muscle. No underlying bony abnormality was seen (Figure 1).

The psoas abscess was surgically drained and the pus from it grew $S$. aureus with identical antibiotic sensitivities to the organism grown from blood culture and the tip of the subclavian venous catheter. Her condition deteriorated postoperatively and she died 2 weeks later, 4 months after her overdose.

\section{Discussion}

There has been no previous report on psoas abscess as a complication of subclavian venous catheterization. Indwelling venous catheters are an important cause of hospital-acquired septicaemia. Strict aseptic techniques should be observed. If regular ob- servation reveals signs of local sepsis, catheters should be removed and the tips sent for culture. In all cases, they ought to be removed within 96 hours. ${ }^{2}$

Psoas abscess is an occult infection and a delay in diagnosis is common. Abdominal CT scan offers the possibility of early diagnosis and is most useful in follow-up. Ultrasonography is also helpful but a small lesion can easily be missed. Gallium scan is frequently positive but results may be delayed up to 72 hours. ${ }^{3}$ Treatment consists of adequate drainage and appropriate antibiotic therapy. Traditionally, drainage was achieved surgically. In recent years, some patients have been successfully treated by percutaneous drainage under the guidance of either ultrasound ${ }^{4}$ or CT scan, ${ }^{5}$ without surgical intervention.

The mortality and morbidity of psoas abscess are significant. ${ }^{3}$ Early diagnosis is the key to satisfactory outcome.

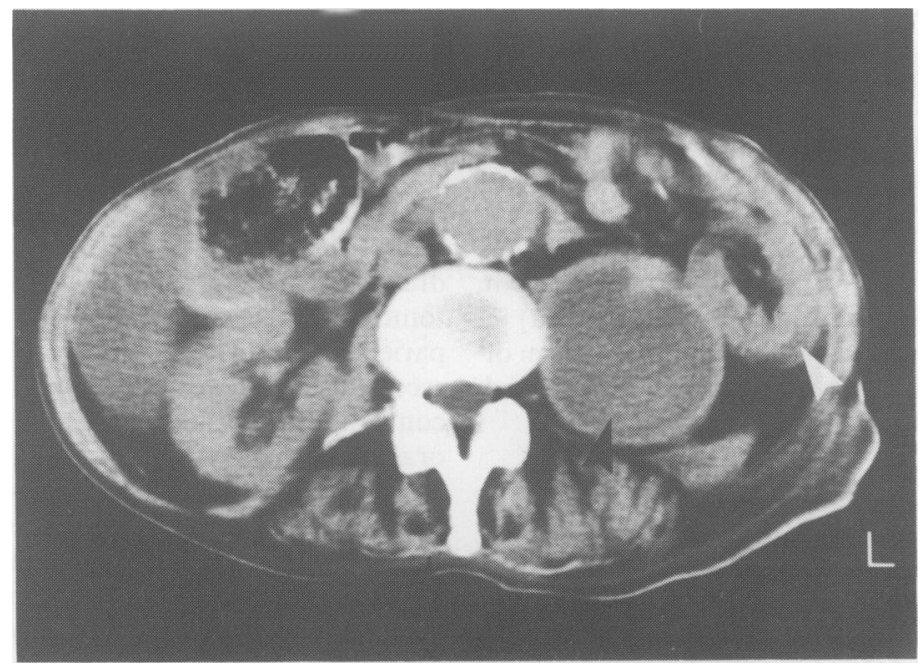

Figure 1 CT scan. Low density area in enlarged left psoas muscle (black arrow). Left kidney (white arrow) displaced laterally. There was no radiological evidence of osteomyelitis.

\section{References}

1. Tillman, B.F., Gibson, R.L. \& Stone, W.J. Psoas abscess in chronic dialysis patients. $J$ Urol 1987, 137: 489-490.

2. Bentley, D.W. \& Lepper, M.H. Septicaemia related to indwelling venous catheter. JAMA 1968, 206: 1749-1752.

3. Gordin, F., Stamler, C. \& Mills, J. Pyogenic psoas abscess: noninvasive diagnostic techniques and review of the literature. Rev Infect Dis 1983, 5: 1003-1011.

4. MacErlean, D.P. \& Gibney, R.G. Radiological management of abdominal abscess. J R Soc Med 1983, 76: 256-261.

5. Vatandaslar, F. \& Alemdaroglu, A. CT-guided percutaneous drainage of psoas abscess. Urology 1987, 29: 450-453. 